Revista de Investigación Educativa 24

enero-junio, 2017 | ISSN 1870-5308 | Xalapa, Veracruz

Instituto de Investigaciones en Educación | Universidad Veracruzana

\title{
Estudiantes indígenas en el contexto de las desigualdades estructurales
}

Indigenous students in the context of structural inequalities

\author{
Mtra. Alexandra Avena Koenigsberger \\ Doctoranda \\ Universidad Pompeu Fabra de Barcelona, España. \\ alexandra.avena01@estudiant.upf.edu
}

En este artículo pretendo analizar la relación entre el sistema educativo mexicano y las estructuras de inequidad. Para ello, analizaré algunos problemas que enfrentan las estudiantes indígenas al momento de hacer efectivo su derecho a la educación. Ofrezco una serie de motivos que explican las desigualdades educativas entre estudiantes indígenas y no indígenas. Para ello, recurro al concepto de desigualdad estructural y argumento que las poblaciones indígenas son víctimas de este tipo de desigualdad. Retomando debates críticos del liberalismo igualitario, finalmente, sugiero que el sistema educativo mexicano debe adoptar los principios de las políticas de la diferencia y, como consecuencia, del multiculturalismo.

Palabras clave: Justicia social; igualdad de oportunidades; multiculturalismo; estudiantes indígenas; educación intercultural.

In this article I intend to analyze the relation between the Mexican educational system and structural inequalities. For that, I will analyze the problems that indigenous students face when trying to make effective their right to education. I offer a series of reasons that explain the educational inequalities between indigenous and non-indigenous students. By developing the concept of structural inequality, I argue that indigenous peoples in Mexico are victims of these type of inequalities. And finally, by drawing on some critical debates against liberal egalitarianism I suggest that the Mexican educational system ought to adopt principles of the politics of difference and, as a consequence, of multiculturalism.

Keywords: Social justice; equality of opportunities; multiculturalism; indigenous students; intercultural education. 


\section{Estudiantes indígenas en el contexto de las desigualdades estructurales}

\section{Indigenous students in the context of structural inequalities}

\section{Introducción}

A ctualmente se reconoce a la educación como un derecho humano. Así lo estaconstituciones de los Estados occidentales.

En efecto, desde la Declaración Universal de Derechos Humanos del 10 de diciembre de 1948 se reconoce, por primera vez, el derecho a la educación en el artículo 26. Es a partir de entonces que surge un entramado de instrumentos jurídicos y de constituciones nacionales que reconocen bajo estos términos, o similares a éste, el derecho a la educación.

En México el derecho a la educación está reconocido en el artículo $3^{\circ}$ de la Constitución Política de los Estados Unidos Mexicanos (CPEUM), indicando que

todo individuo tiene derecho a recibir educación. El Estado - Federación, Estados, Ciudad de México y Municipios—, impartirá educación preescolar, primaria, secundaria y media superior. La educación preescolar, primaria y secundaria conforman la educación básica; ésta y la media superior serán obligatorias.

Y es que, más allá de que la educación ha sido reconocida formalmente como un derecho humano y, como tal, debe ser garantizada a todas las ciudadanas, ${ }^{2}$ el papel que juega la educación formal es relevante por la dimensión preparatoria que la acom-

1. Declaración Universal de los Derechos Humanos; Pacto Internacional de Derechos Económicos, Sociales y Culturales; Convención sobre los Derechos del Niño, artículos 28 y 29; Protocolo de San Salvador sobre Derechos Económicos, Sociales y Culturales, Artículo 13; Protocolo Adicional 1 al Convenio Europeo para la Protección de los Derechos Humanos y de las Libertades Fundamentales, Artículo 2, entre otros.

2. Ya que las mujeres constituimos una mayoría en cuanto a población mundial y atendiendo a una reivindicación de perspectiva de género, estaré refiriéndome en femenino, sin que ello implique que me estoy refiriendo sólo a mujeres ni que este trabajo intenta abordar debates de género. 
paña: amplía oportunidades de vida. Sin que sea el único factor que determina las oportunidades de vida de las ciudadanas, una educación formal contribuye a que las personas puedan perseguir mayores oportunidades económicas, sociales, culturales, profesionales, entre otras (Macleod, 2009).

No obstante, aun cuando la educación básica y media superior son consideradas no sólo como un derecho, sino también como una obligación —-tanto para los padres o tutores, como para el Estado mexicano en cuanto a la obligación de otorgarlo- ${ }^{3}$ los datos que a continuación proporcionaré no permiten afirmar que el derecho a la educación en México sea garantizado a todas las personas. Por ejemplo, la Ciudad de México tiene el promedio más alto en cuanto a escolaridad, siendo éste primero de preparatoria, mientras que Chiapas y Oaxaca se encuentran en el último puesto, al tener una escolaridad promedio de sexto grado de primaria. A nivel nacional, el grado escolar cursado en promedio es segundo año de secundaria (Instituto Nacional de Estadística y Geografía [INEGI], 2010). Esto quiere decir que el promedio de escolaridad a nivel nacional no es superior a la educación básica, y que este nivel escolar sólo ha sido superado en la Ciudad de México.

Estos datos pueden sugerir que no todas las personas tienen las condiciones para acceder a los sistemas educativos estatales. No obstante, hay muchos motivos por los cuales sucede esto, y hacer una afirmación generalizada no permite detectar las principales causas de la falta de acceso a dichos sistemas. Por ello, en este artículo me interesa enfocarme en grupos de personas que no acceden a los sistemas educativos debido a las desigualdades estructurales que enfrentan, principalmente, por su pertenencia a ciertos grupos sociales vulnerables. Para ello, me enfocaré más concretamente en el caso de las poblaciones indígenas.

Sin ignorar que dentro de las demandas de las poblaciones indígenas se encuentra la de controlar sus propios sistemas educativos, y que dentro de estas demandas existe ya amplia literatura sobre la educación intercultural y bilingüe y educación indígena, ${ }^{4}$ la situación de las poblaciones indígenas no se puede englobar en su totalidad dentro de estas demandas. Así, aun cuando la autonomía para controlar sus propios sistemas educativos debe ser seriamente analizada y debatida, en este artí-

3. Sobre los alcances del derecho a la educación en México, ver Valadés (1997) y Latapí (2009).

4. Sobre este tema hay extensa literatura en México. Dentro de ésta destacan, entre otros, Baronnet (2015), Comboni (2002), Lizama-Quijano (2010), Schmelkes (2004), Sartorello (2014) y Bertely (2009). 
culo me centraré en mostrar cómo no existen las condiciones institucionales para que las ciudadanas indígenas que deseen acceder a los sistemas educativos estatales $y$, con ello, poder optar por un abanico de oportunidades de vida distintas a las ofrecidas por su cultura y comunidad, puedan hacerlo en igualdad de condiciones que las personas no indígenas.

Para ello, me enfocaré en la relación que hay entre el sistema educativo estatal y las estructuras de inequidad, e intentaré mostrar cómo las poblaciones indígenas, al ser víctimas de dichas estructuras de inequidad, son afectadas directamente para poder acceder a, y permanecer en, los sistemas educativos estatales.

Sin que sea el único factor que explica la dominación de los pueblos indígenas, el sistema educativo contribuye de manera importante a esta situación. Por ello, sugeriré que es necesario replantear los principios que deben guiar al sistema educativo estatal, con la finalidad de que responda a la diversidad cultural que existe en el país.

\section{Inequidades sociales. Un breve panorama}

Una de las formas más simples para evaluar las desigualdades es a partir de conocer los niveles de pobreza que tiene un país. En el caso de México, para 2012 había 53.3 millones de personas en situación de pobreza, esto equivale a $45 \%$ de la población. Dentro de esa cifra, $10 \%$ de personas se encontraba en situación de pobreza extrema (Consejo Nacional de Evaluación de la Política de Desarrollo Social [CONEval], 2012).

Además, para ese mismo año se estimó que $20 \%$ de la población mexicana (cerca de 24 millones de personas) tenía ingresos inferiores a la línea de bienestar mínimo, ${ }^{5}$ y 51.6\% (60.6 millones de personas) tenía ingresos inferiores a la línea de bienestar. En contraste, para 2013 hubo 15 mexicanos dentro de la lista de los hombres más ricos del mundo que publica la revista Forbes.

Para el periodo 2008-2009, se estimó que 25\% de niños y niñas en México (aproximadamente 10 millones) vivían en una situación de pobreza alimentaria (Fondo de las Naciones Unidas para la Infancia México [UNICEF], 2010). En ese mismo periodo, se estimó que $25.5 \%$ de niños y adolescentes no tenía acceso a la canasta alimentaria

5. La línea de bienestar mínimo equivale a la canasta alimentaria, mientras que la línea de bienestar equivale a la canasta alimentaria más la canasta no alimentaria, la cual incluye transporte público, limpieza y cuidados de la casa, limpieza personal, educación, vivienda, cuidados de la salud, entre otros. Las cantidades son consideradas mensualmente por persona. 
básica, 34\% sufría carencias en salud y educación y 59.5\% no tenía acceso a vivienda, transporte y vestido básicos.

Por otra parte, mientras que en entidades federativas como la Ciudad de México o Nuevo León el Índice de Desarrollo Humano (IDH) es comparable con el de países como Argentina, el IDH de Chiapas es comparable con el de Nicaragua o Siria. Más aún, en municipios de Oaxaca o Chiapas, el IDH es comparable a Senegal o Nigeria, y es justo en estos dos estados de la República donde se concentra el mayor número de poblaciones indígenas del país (Comisión Nacional para el Desarrollo de los Pueblos Indígenas [CDI], 2015).

Por lo que hace a esta población, $53 \%$ de las viviendas indígenas están construidas a base de suelo de tierra; $42.3 \%$ no cuenta con agua entubada en su vivienda; $73 \%$ no cuenta con sistema de drenaje; $20.7 \%$ no cuenta con electricidad, y $13 \%$ no cuenta con agua entubada, sistema de drenaje ni electricidad en su vivienda (CDI, 2015).

En cuanto a los ingresos que reciben las poblaciones indígenas, $25.8 \%$ no recibe ingresos; $27.1 \%$ recibe menos de un salario mínimo; $27.9 \%$ recibe de 1 a 2 salarios mínimos, y 19.1\% recibe más de dos salarios mínimos. Cabe destacar, sin embargo, que de acuerdo con el Índice Nacional de Precios al Consumidor (2016), la canasta básica alimentaria cuesta 2.5 salarios mínimos, lo que nos permite inferir de los datos antes mostrados que sólo 19.1\% de la población indígena puede acceder a ella (CDI, 2015).

Por último, en cuanto a niveles de escolaridad, que es lo que interesa en este artículo, $25.8 \%$ de ciudadanas indígenas no cuenta con instrucción escolar; $27.2 \%$ tiene estudios incompletos del nivel primaria; $18.7 \%$ ha concluido el nivel de primaria, y 28.2\% tiene estudios posteriores al nivel de primaria (CDI, 2015).

De las cifras anteriores es posible afirmar que la situación que viven las poblaciones indígenas no es la adecuada para que las ciudadanas indígenas puedan perseguir sus proyectos de vida o, al menos, puedan optar por perseguir sus formas de vida conforme a su propia concepción de vida buena. Esto es, que la situación que viven las poblaciones indígenas limita su abanico de oportunidades de vida. Más aún, si la educación es un bien posicional que contribuye a que las ciudadanas puedan optar por diferentes proyectos de vida, resulta esencial que ésta esté al alcance efectivo de todas las personas, independientemente de su membresía a grupos culturales, sociales o vulnerables.

Y aunque es verdad que muchas ciudadanas indígenas no acceden a los sistemas escolares estatales porque quizá opten por acceder a sus propios sistemas educativos, ello no implica que el Estado está exento de ofrecer las condiciones óptimas para que aquellas ciudadanas indígenas que quieran acceder a los sistemas escolares estatales 
puedan hacerlo, sin que ello implique un costo que muchos otros sectores de la población no se ven obligados a pagar, como ocurre con las barreras estructurales a las que más adelante me referiré.

Sobre cómo lograr una equidad en las oportunidades educativas de las ciudadanas mucho se ha analizado desde la filosofía política; no obstante, estas teorías dejan de lado factores externos al sistema educativo y, con ello, se convierten en debates que no resultan adecuados para las situaciones reales no-ideales. A continuación mencionaré algunas ideas de este debate.

\subsection{Inequidades educativas. Un breve panorama sobre el debate teórico}

Existen inequidades entre todas las personas y grupos de personas de distintas sociedades. Aun cuando no todas las inequidades son injustas, el interés por eliminar estas desigualdades —injustas — ha acaparado la atención de muchas personas en las últimas décadas. Desde un ámbito educativo ello no es la excepción.

Las inequidades educativas se reflejan en muchos aspectos, como las disparidades en el presupuesto que reciben distintas escuelas de distintas regiones de cada estado, la calidad de los educadores, recursos escolares (libros, laboratorios, comedores, computadoras), número de estudiantes por salón, etcétera (Satz, 2007).

Debido a que, como he mencionado anteriormente, una educación formal es un factor importante al momento de perseguir ciertas formas de vida, ésta debe estar al alcance de todas las ciudadanas en igualdad de condiciones. Por ello, algunas teóricas igualitarias han tratado de dar respuestas a cómo alcanzar justicia en la educación para que todas las personas puedan acceder a la misma y, con ello, puedan optar por las oportunidades de vida que cada una elija.

Desde una perspectiva de la filosofía política contemporánea, Gutmann (2001) fue una de las primeras en analizar los principios que deben guiar a la educación formal. Para ello, defendía "el principio de no discriminación en la educación”, en donde el sistema educativo debe estar abierto a todas aquellas personas que quieran y puedan aprender. En términos similares, Rawls (1999) defiende que todas las posiciones sociales deben estar abiertas para todas las personas, y que la selección para ocupar esas posiciones sociales deberá depender de cuestiones ajenas a raza, clase social, sexo, religión, entre otros.

Cómo entender la igualdad de oportunidades educativas es algo que ha generado mucho debate y muchos puntos de vista encontrados. Por ejemplo, Brighouse y Swift 
(2009) sugieren que la igualdad de oportunidades educativas debe ser entendida de una manera formal: por medio de un parámetro comparativo importa que todas las personas tengan lo mismo. Es decir, importa cuánto tiene una persona con respecto a la otra (Satz, 2007). Para ello, se requiere de un campo de juego nivelado en donde todas las estudiantes tengan la misma oportunidad para competir por el éxito. Pero este campo de juego solamente estará nivelado si son las diferencias en los talentos y habilidades de las estudiantes las que determinen su futuro; sólo de esta manera obtendrán lo que merecen (Swift, 2003).

En opinión de Swift (2003), esta visión meritocrática se basa en que las oportunidades de toda persona de tener una buena educación o tan siquiera de tener una educación y, con ello, seguir abriendo su camino para una vida buena — según su propia concepción del bien-, no deben depender de las capacidades económicas de sus padres ni de otros factores ajenos a ellos, sino que deberían depender de su inteligencia y esfuerzo para acceder al nivel y calidad educativa que desean.

Sin embargo, la teoría del mérito - educativo y no- sugiere una serie de objeciones que hacen cuestionable esta tendencia. Según Rawls (1999), por ejemplo, usar los talentos naturales como un criterio de selección para el acceso a universidades, sería igual de justo que seleccionar bajo un criterio de raza o sexo, porque las personas son igualmente responsables tanto por sus talentos innatos como por su raza o sexo. Por ello, sugiere que el criterio para medir el mérito debería basarse no tanto en cuestiones como el talento innato, que no depende de cada persona, sino del esfuerzo y logros obtenidos.

Según Young (1990), usar el principio del mérito para seleccionar posiciones en una jerarquía laboral o en las instituciones educativas sería justo sólo si reúne ciertas condiciones, principalmente, que las cualidades y los parámetros utilizados para medir el desempeño de las candidatas sean neutrales y estén libres de cualquier contenido o valor cultural.

Pero esta idea de neutralidad, como más adelante mencionaré, es imposible de reunir porque una aparente neutralidad estará, invariablemente, favoreciendo siempre a la cultura mayoritaria. Y esto es fundamental cuando estamos frente a una sociedad culturalmente diversa, como la mexicana.

Como se puede ver, pues, estas discusiones dejan de lado factores externos al sistema educativo y a la forma como están conformadas la mayoría de las sociedades actuales. Por ello, resulta relevante analizar la crítica que hizo Young a estas teorías de igualdad de oportunidades, quien argumentó que serán adecuadas sólo cuando 
logremos tener una sociedad que cuente con una repartición equilibrada y justa de recursos, pero sobre todo, una sociedad libre de relaciones de dominación. No obstante, dado que en nuestras sociedades existen grandes problemas de desigualdad e injusticia social, y de relaciones de dominación que se han ido reforzando a lo largo de la historia, estas teorías resultan interesantes desde un punto de vista académico y abstracto pero poco pueden ofrecer para lidiar con los problemas de fondo y, más concretamente, con las estructuras de inequidad.

En el próximo apartado intentaré mostrar qué se entiende por estructuras de inequidad, cómo éstas se ven reflejadas en el sistema educativo y, finalmente, cómo los pueblos indígenas son una de las principales víctimas de estas estructuras en el contexto de la educación formal.

\section{Educación y estructuras de inequidad}

He venido argumentando que la educación formal es un instrumento importante en la tarea de igualar y expandir todo tipo de oportunidades de vida a todas las personas (Eisenberg, 2006). Es por ello que, como se ha venido insistiendo, una equidad educativa resulta fundamental en una sociedad que aspira a la igualdad y a la justicia, objetivos ambos que, como hemos visto al inicio, buscan algunos de los textos legales que reconocen el derecho a la educación en México.

Aun así, dicha equidad no implica, o no debería implicar, que todas las educandas tengan los mismos resultados educativos cuando egresan de las escuelas, porque cada persona tiene una distinta concepción del bien y de sus proyectos de vida, pero sí debe busca eliminar las injusticias y arbitrariedades que impiden que todas las educandas puedan acceder a las oportunidades educativas que les ayuden a la realización de su potencial (Eisenberg, 2006).

No obstante, una igualdad de oportunidades educativas ya supone un reto difícil. Ello, porque hay muchos obstáculos que impiden que todas las personas puedan hacer efectivos sus derechos como ciudadanas. Parte del problema es que aun cuando hay un reconocimiento formal del derecho de la educación a todas las personas, todavía existen muchas relaciones de dominación y opresión que impiden que éste sea ejercitado en igualdad de condiciones. ${ }^{6}$ Para desarrollar mejor esta idea, a continuación

6. Según Young (1990), la opresión que tenemos hoy en día en nuestra sociedad es distinta de la 
explicaré el concepto de estructuras de inequidad y, posteriormente, intentaré mostrar de qué forma éstas repercuten en las oportunidades educativas de las estudiantes indígenas, de acuerdo a cómo está conformado el sistema educativo mexicano.

\subsection{Sistema educativo y estructuras de inequidad}

Young (2001) teorizó el concepto de estructura de inequidad, entendiendo por éste un conjunto de procesos sociales que, al reproducirse, se refuerzan de tal manera que permiten o limitan la actuación de las personas en distintas maneras.

De modo más preciso, por estructura se entiende el hecho de que las distintas posiciones sociales condicionan las oportunidades y los prospectos de vida de las personas que se encuentran en una determinada posición social, y cuya reproducción fortalece y reafirma a las mismas estructuras. Por ejemplo, la gente perteneciente a una clase alta procura normalmente que sus hijas puedan mantener esa posición social. Por ello, procurarán un ambiente donde todas las expectativas de lo que implica pertenecer a una clase alta (incluida la asistencia a colegios destinados para esa clase) puedan ser alcanzadas. Así, probablemente las hijas de la gente que proviene de una clase alta mantendrán ese estatus y, por tanto, en un futuro serán quienes mantengan grandes relaciones de poder y de influencia. De esta forma, la reproducción continuada de esas expectativas genera que las mismas se vayan reafirmando y normalizando y que, por tanto, el actuar de las personas se vea condicionado a su posición social.

De esta manera, por estructuras de inequidad se entienden las restricciones que algunas personas enfrentan en su libertad y bienestar material como consecuencia de su pertenencia a una posición social determinada, que no necesariamente sufren otras personas pertenecientes a otra posición social, al tener más oportunidades o más fácil acceso a algunos beneficios. Si bien es cierto que estas restricciones no determinan necesariamente el futuro de las personas para lograr sus planes de vida y acceder a beneficios, si hace que poder lograrlo les sea más difícil que a otras que se encuentran en una posición social más ventajosa (Young, 2001).

Estas estructuras de inequidad pueden ser calificadas como injustas cuando el grupo de personas pertenecientes a la posición social menos ventajosa, de entre aqué-

opresión que existía siglos atrás. Para ella, actualmente la sociedad enfrenta cinco caras de opresión: Explotación, Marginación, Falta de Poder, Imperialismo Cultural y Violencia. Por cuestiones de espacio y de objetivos, en este artículo no es posible detallar este tema. 
llas que estamos comparando, guardan un conjunto de características en común que nos permite contar una historia plausible de por qué unos están en ventaja y otros en desventaja (como raza, sexo, religión, pertenecía cultural, entre otras). Así, podemos sugerir que la desigualdad entre dos personas es efectivamente injusta — pues depende de la posición social a la que pertenecen - y no simplemente accidental, o por mala suerte de uno y buena suerte de otro. Es decir, que las inequidades entre personas en sí no son necesariamente injustas y de hecho son bastante normales. Sin embargo, cuando esas inequidades se explican, en parte, debido a la pertenencia a distintos grupos sociales, resulta una inequidad injusta porque ésta es un producto social que ha sido causado por una historia de relaciones y que, de no haber sido creada por la sociedad, no existiría.

De esta manera, bajo estos criterios las desigualdades entre dos personas no dependen de su buena o mala suerte, ni de las decisiones que han tomado a lo largo de sus vidas, sino de una creación social que distingue, en concordancia con una historia plausible, a unas personas de otras. Además, siguiendo a los principios del liberalismo igualitario, las personas no son responsables de sus características innatas, por lo que no deben sufrir ventaja o desventaja sobre estos hechos fortuitos. En ese sentido, nadie decide en qué grupo social nacer $y$, por tanto, hacer una distinción entre personas pertenecientes a dos grupos sociales diferentes resulta injusto a la luz de dichos principios.

Este concepto de estructuras de inequidad resulta relevante para analizar los problemas que enfrenta el sistema educativo mexicano porque, como se ha podido mostrar por las cifras antes citadas, no todas las personas en México gozan de los mínimos básicos de subsistencia, y una gran mayoría de ciudadanas indígenas no cuenta con los recursos para poder, siquiera, acceder a una canasta básica alimentaria. Por ejemplo, $87.9 \%$ de la población indígena vive debajo de los niveles de pobreza (Schmelkes, 2013), y en un estudio realizado en 2006, 32\% de niñas indígenas menores de 5 años medía menos que el promedio para esa edad. Además, la tasa de mortalidad en niñas indígenas era 60\% mayor que en niñas no indígenas, lo cual muestra los problemas de desnutrición y falta de salud que padecen estas poblaciones, debido, en parte, a los ingresos que reciben las familias indígenas (UNICEF México, 2014).

Esta desnutrición, en línea con Schmelkes (2013), se manifiesta en el hecho de que en los procesos de aprendizaje las niñas indígenas tardan casi el doble que las no indígenas en aprender a leer y escribir. De hecho, $14 \%$ de niñas indígenas de entre ocho y catorce años que asisten a la escuela son analfabetas, mientras que sólo $2.4 \%$ de niñas no indígenas que asiste a la escuela están en esa misma situación. 
Pero existen aún más desigualdades entre indígenas y no indígenas en los centros educativos. Por ejemplo, un estudio realizado por el Instituto Nacional para la Evaluación en la Educación (INEE, 2016) reveló que mientras que 22.8\% de estudiantes indígenas afirmó no contar con un solo libro en su casa, sólo $11 \%$ de estudiantes no indígenas afirmó estar en esa misma situación; además, $13 \%$ de estudiantes indígenas dijo contar con computadora en su casa, mientras que $40 \%$ de estudiantes no indígenas dijo contar con computadora.

En cuanto a la ayuda recibida en su casa para poder realizar los deberes escolares, de las estudiantes indígenas encuestadas $31 \%$ dijo que nunca recibe ayuda; $27 \%$ dijo que a veces recibe ayuda; $24 \%$ dijo que casi siempre, y $13 \%$ dijo que siempre recibe ayuda. A su vez, $15 \%$ de las estudiantes no indígenas señaló que nunca recibe ayuda en su casa; $21 \%$ dijo que a veces; $40 \%$ dijo que casi siempre, y $24 \%$ dijo que siempre recibe ayuda en su casa.

Ante estos factores de inequidad entre estudiantes indígenas y no indígenas $-\mathrm{y}$ es necesario tener en cuenta que ahora ya estoy refiriéndome a estudiantes indígenas que asisten a los centros educativos estatales y, por tanto, ya no me estoy refiriendo al sector de poblaciones indígenas que demandan autonomía para poder impartir sus propios sistemas educativos - resulta evidente que es problemático aplicar los mismos procesos de aprendizaje, así como los mismos estándares de evaluación a todas las estudiantes por igual. Es decir, que si hay tanta disparidad en los factores socioeconómicos entre indígenas y no indígenas, al aplicar un trato igualitario a todas las estudiantes sin distinción alguna, las probabilidades de que las estudiantes indígenas sean las menos exitosas educativamente son altas. De hecho, tal como afirma otro estudio realizado por el INEE, son las estudiantes indígenas las que peores resultados académicos obtienen (INEE, 2006; Schmelkes, 2004).

Podemos ver, entonces, cómo las poblaciones indígenas son víctimas de las estructuras de inequidad que ya he analizado anteriormente. Cuando el nivel y la calidad de la educación que recibe cada niña se ven influidos por el nivel socioeconómico de sus padres, o al menos, de la posición social en la que se encuentran, podemos ver que las poblaciones indígenas tienen las menores posibilidades de completar exitosamente los estudios formales y, aun cuando logren terminar de manera exitosa éstos, el trabajo y el esfuerzo que habrán tenido que realizar ellas y sus familias habrán sido muy superiores a los de cualquier otra estudiante que no se encuentra en esa misma situación.

Actualmente, en muchas sociedades el garantizar una educación de calidad es responsabilidad principalmente de los padres y madres y no de la sociedad, comuni- 
dad o Estado (Young, 2006), lo que sin duda retroalimenta las estructuras de inequidad, porque no todas las familias pueden enviar a sus hijos a las escuelas, o a mejores escuelas, y no todas las familias pueden proveer de las condiciones más óptimas a sus hijos para poder obtener las mejores notas en las escuelas. Este sistema, bajo estas condiciones, sumadas a las que posteriormente se expondrán, en vez de romper las estructuras de inequidad las refuerza, porque un niña que proviene de una familia indígena tendrá mayores posibilidades de repetir el patrón de sus padres, y menores posibilidades de abrir su abanico de oportunidades de vida y poder optar por otra forma de vida, si así lo quisiera.

Debido a la existencia de inequidades estructurales en México, un bien básico como lo es la educación que, aparentemente, está garantizado a todas las personas - ya que no le prohíbe explícitamente a una persona o a un grupo de personas recibir este beneficio- en la realidad sólo puede ser gozado por quienes pertenecen a las posiciones sociales más ventajosas, puesto que personas pertenecientes a otras posiciones sociales encontrarán mayores obstáculos tanto en el acceso como en su permanencia en los sistemas educativos.

Con esto, se puede ver que el principio igualitario que rige al sistema educativo mexicano - y que más adelante volveré a analizar más detalladamente-, aun cuando es garantizado formalmente a todas las personas, puede resultar excluyente (Carnoy, 1997). Sólo quienes reúnan ciertas circunstancias o condiciones podrán acceder al mismo o, al menos, a los niveles más elevados y de mejor calidad, creando una distinción entre diferentes grupos de personas (las educadas y las no educadas), en donde la pertenencia a los distintos grupos sociales juega un papel importante, y es por este motivo que esta distinción resulta injusta.

Pero además, y como resultado de lo anterior, ya desde el hecho de que unas acceden al sistema educativo y otras no, sobre todo en cuanto a la educación superior que es la que prepara a las estudiantes para ejercer profesiones, es como se lleva a cabo la distinción potencial entre profesionales y no profesionales, que tiene como consecuencia la falta de poder como una forma de opresión. ${ }^{7}$ Esto tendrá grandes y ne-

7. Young (1990) afirma que el actual mercado laboral está dividido entre profesionales y no profesionales. Los primeros son beneficiados en mayor medida por el trabajo que realizan los segundos, los cuales sufren una forma de opresión llamada falta de poder — powerlesness.

De acuerdo con esta forma de opresión, los no profesionales carecen de la autoridad y el estatus que sí gozan los profesionales. El problema de esta división — profesional y no profesional o manual y mental - es que trasciende el ámbito laboral, de forma que es traspasada a la vida diaria. Es decir, que 
gativas repercusiones en el futuro de las ciudadanas, pues serán juzgadas tomando en cuenta la posición social a la que pertenezcan, la cual dependerá en cierta medida del grado de educación adquirido por medio del sistema educativo. En pocas palabras, la situación se torna en algo cíclico, donde resulta complejo detectar un solo problema como la causa de tantas desigualdades sociales; pero sin duda, el sistema educativo juega un papel importante en este bucle, pues la forma como está conformado repercute - o refuerza - las desigualdades injustas.

En resumen, he intentado argumentar y mostrar cómo los pueblos indígenas son víctimas de las desigualdades estructurales. Esto se refleja al momento de intentar acceder y permanecer en los sistemas educativos estatales, porque su posición social los restringe y condiciona a la reproducción de determinados roles sociales, al cumplimiento de ciertas expectativas por su calidad de indígenas, que los coloca en la posición social más marginada de la sociedad mexicana.

Esta vulnerabilidad generalizada en la que se encuentran les afecta directamente en sus posibilidades de acceder a una educación y, con ello, a romper con las estructuras sociales que los condicionan y limitan para poder acceder a otras oportunidades de vida. Si la educación en su dimensión preparatoria es un medio necesario para poder acceder a un amplio abanico de oportunidades de vida, y la población indígena no tiene acceso a ella de la misma manera que tiene acceso una persona no indígena, entonces existe una desigualdad injusta entre quienes son indígenas y quienes no lo son. Esta desigualdad estructural, al reproducirse continuamente y perpetuarse en el tiempo, impide que el grupo social indígena pueda romper las expectativas que de dicho grupo se tiene $y$, por tanto, que puedan acceder a todo tipo de oportunidades de vida.

Además, muchas personas podrán pensar que la situación de las poblaciones indígenas con respecto a los sistemas educativos es así por las demandas que algunas poblaciones indígenas han hecho para controlar sus propios sistemas educativos. Y es verdad que existen poblaciones indígenas que han hecho efectiva esta demanda, pero como insistí anteriormente, no es así para todos los integrantes de este tipo de poblaciones. En este artículo mi interés es en aquéllas estudiantes indígenas que quieren optar por cursar el sistema educativo estatal y que, debido a su posición social, encuentran mayores obstáculos que el resto de las estudiantes no indíge-

los profesionales y no profesionales incluso operan en distintos mundos: viven en barrios separados, asisten a distintas escuelas, sus distinciones incluso recaen en qué comen, qué visten, qué música escuchan, entre otros. Esta distinción, en la mayoría de las ocasiones, ha servido para dividir y discriminar y no para incluir y unir. 
nas. ${ }^{8}$ En estos casos estamos frente a una estructura de inequidad que se ha venido reproduciendo por siglos y que es injusta principalmente porque nadie decide en qué posición social nacer, y porque nadie debería estar predestinado a repetir las expectativas que de esa persona se espera debido al grupo social al que pertenece.

Lo anterior sugiere que el sistema educativo mexicano no contribuye a garantizar que todas las personas puedan acceder a una educación formal en igualdad de condiciones. Esto es, principalmente, porque lo rigen los principios de trato igualitario, neutralidad y no discriminación, que son fundamentales al pensamiento liberal igualitario. No obstante, en el siguiente apartado intentaré argumentar cómo estos principios deben ser abandonados al no ser capaces de lidiar con ciertas injusticias sociales y con las inequidades estructurales.

Estos principios liberales igualitarios los encontramos reflejados en el mérito académico. A continuación mostraré los problemas que surgen cuando este criterio es el que rige al sistema educativo.

\section{El problema del mérito académico}

Actualmente en la mayoría de los sistemas educativos el criterio meritocrático juega un papel fundamental. Mediante éste se pretende determinar los resultados educativos, las cuales juegan un papel importante al momento de ofrecer o negar distintas y variadas oportunidades de vida a los y las educandas.

El problema de utilizar el mérito académico de esta forma puede resultar problemático, pues al aspirar a ser neutral en cuanto al contenido cultural — tanto en el currículo académico como en el proceso evaluativo- e igual hacia todas las estudiantes, su consecuencia puede ser la exclusión de aquéllas que no se adaptan o que no adoptan las pautas de la cultura dominante. Tal es el caso de las estudiantes indígenas.

8. Esto no implica que en los currículos académicos no se considere la pluriculturalidad de la sociedad mexicana. Como mencionaré adelante, existe una obligación del Estado mexicano de promover una educación multicultural, respetuosa con la diversidad tanto de la sociedad mexicana como de las estudiantes que convergen en los mismos centros educativos. El hecho de que ciudadanas indígenas opten por acceder a los sistemas educativos estatales no debe entenderse como que están dispuestas a abandonar su cultura originaria y, por tanto, los currículos educativos deben ser sensibles a la diversidad. 
En efecto, un principio fundamental del liberalismo ha sido la idea de un Estado neutral a todas las formas de vida. Esto implica, dentro de otras cosas, que las instituciones del Estado deben permanecer neutrales tanto a contenidos religiosos como culturales, éticos. Y, a su vez, las instituciones deben ofrecer ciertas condiciones para que todas las ciudadanas puedan ejercer y practicar sus propias formas de vida conforme a su cultura, religión, creencias. Este ideal de neutralidad trae consigo, como corolario, el principio de no discriminación que se ha traducido en un trato igualitario. ${ }^{9}$ Este principio demanda que todas las personas, independientemente de su color de piel, religión, cultura, sexo, deben ser tratadas igual y, más aún, que todas las personas deben ser medidas con los mismos parámetros. Un trato diferenciado, según este principio, resulta discriminatorio y, por tanto, no es compatible con los principios liberales (Barry, 2000).

No obstante, desde una perspectiva crítica del liberalismo igualitario se ha defendido que es imposible que el Estado permanezca neutral a contenidos culturales, pues esta aparente neutralidad en realidad sólo refuerza la cultura dominante a costa de las culturas minoritarias. Es decir, que al no ser posible sostener una neutralidad cultural, el Estado y sus instituciones siempre estarán actuando siguiendo las pautas establecidas por la cultura dominante.

Como consecuencia, el trato igualitario que defiende el liberalismo se convierte en un medio para obligar a las ciudadanas de culturas minoritarias a conformar ciertos estándares que no les son propios. Al ser medidas en función estándares impuestos por la cultura dominante, las ciudadanas pertenecientes a grupos minoritarios se ven obligadas a decidir entre asimilación o exclusión.

El rechazo a la neutralidad del Estado y al trato igualitario es uno de los pilares fundamentales del multiculturalismo. En esta línea, Kymlicka (1995) y Taylor (1994), entre otros, han intentado mostrar cómo esta aparente neutralidad estatal en realidad sólo refuerza las pautas culturales de las culturas dominantes, así como los procesos de exclusión de las personas que son culturalmente distintas. En México, por ejemplo, Ibarra (2005) ha argumentado, siguiendo esta idea, que

9. Donde igualitario implica tratar a todas las personas por igual, sin atender a sus propias diferencias ni desventajas. Se distingue de un trato equitativo, donde éste sí atiende a las diferencias existentes entre ciudadanas y, sobre todo, es sensible a las distintas desigualdades sociales. Esta distinción también se conoce como igualdad formal e igualdad sustanciosa. En pocas palabras, el trato igualitario intenta medir a todas las personas bajo los mismos parámetros. Sobre este debate, ver Barry (2000), Kymlicka (2001), Taylor (1994) y Young (1990). 
si la lengua oficial es la que se utiliza tanto para la economía como para las funciones del Estado, es evidente que aquellos grupos o personas que hablen ese idioma y compartan esa cultura poseerán una ventaja significativa con relación a aquellos que no la hablen. Lo anterior implica que las minorías enfrentan una severa discapacidad frente a tal proceso de construcción del Estado nacional, pues no tendrán la capacidad de proteger su cultura ante una cultura dominante y fuertemente promovida por el propio Estado. (p. 96)

Así, estos teóricos sugieren que el Estado debe abandonar la idea de neutralidad y adoptar una actitud activa que sirva para promover las distintas culturas existentes dentro de su territorio. Esta idea, fuertemente defendida por el multiculturalismo, argumenta que al no existir una neutralidad cultural, el Estado y sus instituciones no deben aparentar ser neutrales $y$, de hecho, deben jugar un papel activo que sirva para promover las condiciones de igualdad entre las distintas culturas. Esto implica políticas multiculturales que intentan igualar la situación de todas las culturas existentes dentro del Estado. Sobre todo, implica que los distintos grupos culturales perciban que el Estado y sus instituciones les pertenecen por igual y que éste no sólo pertenece a la cultura dominante (Kymlicka, 1995, 2003).

Como consecuencia, el trato igualitario debe ser reemplazado por un trato diferenciado que sea capaz de atender a las ciudadanas respetando sus diferencias culturales, con el fin de que puedan hacer efectivos sus derechos como ciudadanas sin tener que abandonar su entorno cultural y su identidad.

En este contexto, el mérito académico resulta ser una evaluación aparentemente objetiva y neutral que mide, sin discriminación, el aprovechamiento educativo de las estudiantes. Esto significa que el criterio del mérito es la aplicación de los principios liberales igualitarios al campo educativo. Al carecer de tratos diferenciados hacia estudiantes culturalmente diversas, como lo son las estudiantes indígenas, éstas se ven orilladas a elegir entre asimilarse a las pautas de la cultura dominante o aferrarse a sus pautas culturales e identitarias y, como consecuencia, permanecer excluidas.

En este sentido, los estudios dirigidos por UNICEF México, mostraron que las principales causas de abandono entre las estudiantes indígenas son la falta de motivación al no poder avanzar de igual forma que sus compañeras no indígenas, y la percepción de no ser respetadas en cuanto a su identidad indígena y a sus diferencias culturales (UNICEF México, 2014). Esto contrasta positivamente con el hecho de que son las estudiantes indígenas las que en promedio obtienen peores notas (INEE, 2006; Schmelkes, 2013). 
Aun cuando hay una falta de datos en cuanto a este punto (Schmelkes, 2013), es posible pensar que, además de las desventajas que existen entre estudiantes indígenas y no indígenas en su entorno familiar, el hecho de que durante los procesos de aprendizaje y en el contenido del currículum académico no se consideran las diferencias culturales afecta negativamente a las estudiantes indígenas. Esto genera una falta de motivación, un profundo daño en su identidad y una percepción de ser excluidas debido a sus diferencias (Taylor, 1994). ${ }^{10}$ Se refuerza, entonces, la idea de "los otros", lo que contribuye al abandono escolar por parte de las estudiantes indígenas.

Así, el hecho de que el sistema educativo gire en torno a un criterio meritocrático que es aplicado en términos iguales a todas las estudiantes, sin considerar sus diferencias culturales, refuerza las inequidades entre estudiantes $y$, sobre todo, refuerza las estructuras de inequidad que hacen que, en primer término, sea difícil que las estudiantes indígenas puedan competir en igualdad de condiciones con las no indígenas.

En distintos países que han reconocido su carácter multicultural se han implementado distintos modelos educativos que intentan lidiar con las diferencias culturales (McAndrew, 2013; Banks, 2004) y, sobre todo, que intentan lidiar con el dilema que se presenta en el campo educativo: la unidad nacional sin asimilación y el respeto a la diferencia sin fragmentación — o como lo ha señalado Banks (2004), entre unidad y diversidad. Cómo lograr esta unión entre diversidad y unidad en el campo educativo es trabajo de las educadoras y pedagogas, pero para ello es necesario primero que el Estado y sus instituciones educativas adopten unos principios más acordes con las necesidades y realidades, tanto sociales como culturales, de México.

Esto da pie a analizar los objetivos que se buscan con la educación formal, lo cual será el último punto de análisis de este artículo.

10. Sobre este punto, Taylor (1994) señala que una falta de reconocimiento hacia las identidades culturales de miembros de grupos minoritarios puede representar un daño personal tan profundo al grado puede desembocar en una forma de opresión. 


\section{El sistema educativo mexicano y sus fines. Una educación para la democracia}

Según el artículo 3 de la Constitución Política de los Estados Unidos Mexicanos, el sistema educativo debe estar encaminado, principalmente, a desarrollar los valores y virtudes democráticas de las ciudadanas. En efecto, dicho artículo menciona que el sistema educativo debe contribuir a "desarrollar armónicamente las facultades del ser humano y [debe fomentar] en él, a la vez, el amor a la Patria, el respeto a los derechos humanos y la conciencia”. Además, la educación ofrecida debe seguir los siguientes criterios:

a. Será democrática, considerando a la democracia no solamente como una estructura jurídica y un régimen político, sino como un sistema de vida fundado en el constante mejoramiento económico, social y cultural del pueblo;

b. Será nacional, en cuanto — sin hostilidades ni exclusivismos - atenderá a la comprensión de nuestros problemas, al aprovechamiento de nuestros recursos, a la defensa de nuestra independencia política, al aseguramiento de nuestra independencia económica y a la continuidad y acrecentamiento de nuestra cultura;

c. Contribuirá a la mejor convivencia humana, a fin de fortalecer el aprecio y respeto por la diversidad cultural, la dignidad de la persona, la integridad de la familia, la convicción del interés general de la sociedad, los ideales de fraternidad e igualdad de derechos de todos, evitando los privilegios de razas, de religión, de grupos, de sexos o de individuos.

De lo anterior se puede concluir que el sistema educativo mexicano debe intentar desarrollar las virtudes democráticas de las ciudadanas. Y de ser así, los principios que deben guiar a los modelos educativos deben ser consecuentes con estos fines. Es decir, que el sistema educativo tiene una tarea que va más allá de transmitir conocimiento, que consiste en transmitir valores democráticos.

Sobre este tema, Gutmann (2001) insistía en que la educación debe forjar buenas futuras ciudadanas, esto es, cultivar su carácter moral, enseñando la virtud democrática, que implica la habilidad de deliberar y, por tanto, participar en la reproducción social consciente. En consecuencia con esta visión democrática de la educación, el logro educativo no debería medirse por las notas y tampoco por los ingresos obtenidos una vez concluidos los estudios, ni por la posición laboral que ocupan las ciudadanas. Al contrario, el logro educativo debe medirse por la capacidad de deliberar y participar en la política democrática en nuestra sociedad. 
Siguiendo esta tesis democrática de la educación, Anderson (2007) propone cuatro dimensiones que debe satisfacer el sistema educativo: i) conciencia sobre las necesidades y problemas de las personas de todos los sectores sociales; ii) una disposición para servir esos intereses y satisfacer esas necesidades; iii) conocimientos técnicos que permitan mitigar esas necesidades y avanzar en los intereses sociales, y finalmente, iv) interacción respetuosa de todas las personas de distintos sectores sociales.

Si bien la adquisición de conocimientos cognitivos es una dimensión importante de la educación democrática, éste no es el único criterio que se debe evaluar.

Por otro lado, ya que se busca educar para la democracia, el modelo educativo debe considerar el pluralismo existente en la sociedad. Es decir, que una sociedad democrática exige que se dé voz a los distintos grupos sociales y culturales (Young, 2001). En dicha situación, se debe incluir a las poblaciones indígenas, lo que implica no sólo que tengan voz en el diseño de los programas educativos, ni en el diseño de los distintos modelos educativos. Además, implica que dentro de los principios que deben guiar a las políticas educativas se introduzca un principio intercultural, que va más allá de atender las diferencias de las poblaciones culturalmente diversas. Una educación intercultural, siguiendo a Tubino (2005, 2013), debe estar dirigida a todas las educandas y no sólo a las educandas indígenas, pues para terminar con las relaciones de poder y dominación debemos no sólo intentar mejorar la situación de los oprimidos, sino también intentar cambiar la actitud de los opresores.

En esta perspectiva, una educación para la democracia debe ser también intercultural. Pero esta interculturalidad no implica otorgar autonomía a las comunidades indígenas para que éstas tengan el control de sus sistemas educativos - como mencioné previamente, este debate es distinto. En este caso, el principio de interculturalidad en la educación implica considerar las diferencias culturales que existen entre las estudiantes, de tal manera que aquéllas que pertenecen a grupos minoritarios no se sientan excluidas y diferentes.

El principio de interculturalidad en la educación, siguiendo la tesis de Tubino (2013), implica derribar los prejuicios y estereotipos creados en torno a las identidades indígenas. El objetivo debe estar dirigido a ofrecer a todas las estudiantes las mismas oportunidades educativas, sin que ello implique que estudiantes indígenas tengan que renunciar a su identidad y a su cultura para poder optar por otras formas de vida. Educar para la democracia en un Estado que se ha reconocido multicultural implica ofrecer una educación que pueda generar cambios transformadores en donde las re- 
laciones de dominación pasen a ser relaciones de cooperación y de equidad. Para ello, es necesario atender a las diferencias en lugar de ignorarlas.

\section{Conclusiones}

He intentado mostrar, a lo largo de este artículo, la relación que existe entre las estructuras de inequidad y el sistema educativo mexicano. Para ello, he centrado mi análisis en los pueblos indígenas.

Es necesario distinguir las demandas que hacen los pueblos indígenas para tener el control sobre sus sistemas educativos tradicionales de la obligación que tiene el Estado, por medio de sus autoridades educativas, de ofrecer las condiciones para que las estudiantes indígenas que quieran acceder a los sistemas educativos estatales puedan hacerlo sin tener que pagar un costo alto que normalmente es o la asimilación o la exclusión.

Las ciudadanas indígenas son víctimas de las estructuras de inequidad que las restringe y limita a desarrollar un papel determinado dentro de la sociedad. Los obstáculos estructurales que enfrentan para poder acceder y permanecer dentro de los centros educativos estatales son, en términos generales, mayores que los de las estudiantes no indígenas.

Como consecuencia, estos obstáculos estructurales afectan directamente su rendimiento. Muestra de ello es que, por lo general, son las estudiantes indígenas quienes obtienen los peores resultados académicos. Y esto, a su vez, refuerza su situación desventajosa. Así, aun cuando el sistema educativo no es el único responsable de la situación de opresión que viven las ciudadanas indígenas, sí juega un papel determinante.

Para que el sistema educativo ofrezca las condiciones adecuadas para que todas las estudiantes puedan perseguir sus distintas formas de vida, es necesario que se atienda a las diferencias culturales de quienes convergen en los centros educativos. En concreto, es necesario intentar proporcionar igualdad de oportunidades educativas a las estudiantes indígenas sin que esto implique que tienen que renunciar a su identidad y a su cultura. En este sentido, es necesario adoptar los principios de las políticas de la diferencia y rechazar el modelo liberal igualitario de neutralidad e igualdad formal.

Por último, para que la educación impartida por el Estado sea democrática, las poblaciones indígenas deben estar implicadas tanto en los principios y valores que se busca transmitir en los centros educativos, así como en el diseño curricular. 
Dentro de una educación democrática, he argumentado que es necesario atender a un principio de interculturalidad en la educación que intenta transmitir el respeto por las diferentes identidades culturales y, sobre todo, terminar con los estereotipos y con las relaciones de dominación. Para ello, la interculturalidad en la educación no debe ser sólo dirigida a las estudiantes indígenas, sino a todas las estudiantes, pues para terminar con las relaciones de dominación es necesario también cambiar la actitud de los dominantes.

\section{Lista de referencias}

Anderson, E. (julio, 2007). Fair opportunity in education: a democratic equality perspective. Ethics, $117(4), 595-622$.

Banks, J. (Ed.). (2004). Diversity and citizenship education: Global perspectives. Nueva York: John Wiley and Sons.

Baronnet, B. (enero-abril, 2015). Derecho a la educación y autonomía zapatista en Chiapas, México. Convergencia, 22(67), 85-110.

Barry, B. (2000). Culture and equality. An egalitarian critique of multiculturalism. Cambridge Massachusetts: Harvard University Press.

Bertely, M. (Coord.). (2009). Sembrando nuestra propia educación intercultural como derecho. Diálogos, experiencias y modelos educativos de dos organizaciones indígenas del estado de Chiapas. México: Alcatraz.

Brighouse, H., \& Swift, A. (mayo, 2009). Educational Equality versus Educational Adequacy: A critique of Anderson and Satz. Journal of Applied Philosophy, 26(2), 471-479.

Carnoy, M. (1997). La educación como imperialismo cultural. México: Siglo Veintiuno.

Comboni, S. (2002). Interculturalidad, educación y política en América Latina. Política y Cultura, 17, 261-288.

Comisión Nacional para el Desarrollo de los Pueblos Indígenas. (2015). Fichas de Información Básica de los Pueblos Indígenas. Recuperado de http://www.cdi.gob. $\mathrm{mx} /$ indicadores2015/\#/2

Consejo Nacional de Evaluación de la Política de Desarrollo Social. (2012). Anexo estadístico de pobreza en México. Recuperado de http://www.coneval. gob.mx/Medicion/Paginas/Medici\%C3\%B3n/Pobreza\%202012/Anexoestad\% $\mathrm{C}_{3} \%$ ADstico-pobreza-2012.aspx 
Einsenberg, A. (enero, 2006). Education and the politics of difference: Iris Young and the politics of education. Educational Philosophy and Theory, 3o(1), 7-23.

Fondo de las Naciones Unidas para la Infancia México. (2010). Pobreza y desigualdad. Recuperado de http://www.unicef.org/mexico/spanish/17046.htm

Fondo de las Naciones Unidas para la Infancia México. (2014). Buenas prácticas sobre educación indígena. Recuperado de http://www.unicef.org/mexico/spanish/ BP Educacion Indigena.pdf

Gutmann, A. (2001). La educación democrática. Una teoría política de la educación. Barcelona: Paidós.

Ibarra, F. (2005) Minorías Etnoculturales y Estado nacional. México: Universidad Nacional Autónoma de México.

Índice Nacional de Precios al Consumidor. (2016). Canasta básica mexicana 2016. Recuperado de http://elinpc.com.mx/canasta-basica-mexicana/

Instituto Nacional de Estadística y Geografía. (2010). Censo Nacional de Población y Vivienda. Recuperado de http://cuentame.inegi.org.mx/poblacion/escolaridad.aspx?tema $=$ P

Instituto Nacional para la Evaluación en la Educación. (2006). Indicadores del Sistema Educativo Nacional, 2006. Recuperado de http://www.inee.edu.mx/index. $\mathrm{php} /$ bases-de-datos/estandares-nacionales

Instituto Nacional para la Evaluación en la Educación. (2016). Consulta previa, libre e informada a pueblos y comunidades indígenas sobre evaluación educativa. Recuperado de http://www.inee.edu.mx/index.php/558-consulta-a-pueblos-ycomunidades-indigenas

Kymlicka, W. (1995). Multicultural citizenship. A Liberal theory of minority rights. Nueva York: Oxford University Press.

Kymlicka, W. (2001). Politics in the Vernacular. Nationalism, Multiculturalism and Citizenship. Oxford: Oxford University Press.

Kymlicka, W. (2003). Multicultural States and Intercultural Citizens. Theory and Research for Education, 1(2), 147-169.

Latapí, P. (enero-marzo, 2009). El derecho a la educación. Su alcance, exigibilidad y relevancia para la política educativa. Revista Mexicana de Investigación Educativa, 14(40), 255-287.

Lizama, J. (Coord.). (2010). Los caminos de la educación intercultural. Cuatro ensayos. Barcelona: Icaria.

Macleod, C. (2009). The Retreat from Educational Equality. Recuperado de https:// 


\section{papers.ssrn.com $/$ sol3/papers.cfm?abstract $\mathrm{id}=1451925$}

McAndrew, M. (2013). Fragile majorities and education. Belgium, Catalonia, Northern Ireland and Quebec. Montreal y Kingston: McGrill-Queen's University Press.

Rawls, J. (1999). A theory of justice. Cambridge: The Belknap Press of Harvard University Press.

Sartorello, C. (enero-marzo, 2014). La co-teorización intercultural de un modelo curricular en Chiapas, México. Revista Mexicana de Investigación Educativa, 19(60), 73-101.

Satz, D. (julio, 2007). Equality, adequacy, and education for citizenship. Ethics, 117(4), 623-648.

Schmelkes, S. (enero-marzo, 2004). La educación intercultural: un campo en proceso de consolidación. Revista Mexicana de Investigación Educativa, 9(20), 9-13.

Schmelkes, S. (enero-abril, 2013). Educación y pueblos indígenas: problemas de medición. Realidad, Datos y Espacio. Revista Internacional de Estadística y Geografía, 4(1), 5-15. Recuperado de http://www.inegi.org.mx/RDE/RDE_08/Doctos/RDE_08_Art1.pdf

Swift, A. (2003). How not to be a Hypocrite: School Choice for the Morally Perplexed Parent. Londres: Routledge.

Taylor, C. (1994). Multiculturalism and “The politics of recognition”. En A. Gutmann, (Ed.), Multiculturalism and "The politics of recognition" (pp. 25-74). Nueva Jersey: Princeton University Press.

Tubino, F. (julio-diciembre, 2005). La praxis de la interculturalidad en los Estados Nacionales Latinoamericanos. Cuadernos interculturales, 3(5), 83-96.

Tubino, F. (2013). Intercultural practices in Latin American Nation States. Journal of Intercultural Studies, 34(5), 604-619.

Valadés, D. (1997). Derecho a la educación. México: Mc-Graw Hill/Universidad Nacional Autónoma de México.

Young, I. M. (1990). Justice and the politics of difference. Nueva Jersey: Princeton University Press.

Young, I. M. (enero, 2001). Equality of whom? Social groups and judgments of injustice. The Journal of Political Philosophy, 9(1), 1-18.

Young, I. M. (2006). Education in the context of structural injustice: A symposium response. Educational Philosophy and Theory, 38(1), 93-103. 